\title{
Optimization of efficient path selection criteria in urban rail transit passenger assignment
}

\author{
Y. Cheng \& X. F. Ye \\ Key Laboratory of Road and Traffic Engineering of \\ the Ministry of Education, Tongji University, China
}

\begin{abstract}
In urban rail transit systems, passengers take a portion of paths (which are called efficient paths) into consideration and ultimately choose one route from them. The correctness of alternative efficient paths has a direct impact on the final result of the passenger assignment model, which plays a key role in urban rail transit planning and operation. The criteria of path selection mainly influence the validity of efficient paths. In this study, alternative efficient path sets under different criteria are investigated based on the route choices of urban rail transit passengers in Shanghai, China, which is derived from mobile phone data. By changing threshold values, two types of risks are analysed to illustrate the difference between alternative and actual efficient path sets. The paper further proposes two optimization methods with the objectives of minimizing the sum of two types of risks. The first one is adopting threshold combination, while the second one is adjusting the limit of interchange times according to the shortest path's interchange times. Experiments show that no matter how much the limit of interchange times is, the performance of alternative efficient path sets under threshold combination is better than the ones under a single threshold (such as absolute and relative threshold). The result also shows that the second method can obviously prevent inefficient paths from entering alternative sets.

Keywords: urban rail transit, efficient path, sensitivity analysis, two types of risks, threshold combination, interchange times.
\end{abstract}

\section{Introduction}

Passenger assignment result is a crucial reference for passenger flow forecast and ticket income distribution. With the development of urban rail transit, there are 
more and more interchange stations within the network, leading to the increase of optional paths for passengers. To allocate passengers scientifically and rationally, a route choice model should be established.

Since urban rail transit network is strongly connected, theoretically, there are countless paths for passengers to choose from. However, due to excessive travel and interchange times, most of the paths are out of the question. Only small parts of the paths are chosen as options by passengers, which are called "efficient paths". Screening out these paths is the first step of the route choice model. Based on the result, the probability of each path to be chosen can be determined according to their impedance value. Therefore, the number and correctness of efficient paths directly affects the final result of passenger assignment.

The remainder of this paper is outlined as follows: Section 2 reviews the previous literature about efficient path selection criteria. Section 3 develops a sensitivity analysis method to compare threshold values. The results are then given and discussed in Section 4. Section 5 proposes two optimization methods to improve the performance of alternative efficient path sets. Section 6 presents the conclusions.

\section{Literature review}

The study on search method of efficient paths has been comparatively mature. What mainly influences the number and correctness of efficient paths is selection criteria. Tagliacozzo and Pirzio [1] propose that in urban road traffic, the elongation ratio ranges from 1.3 to 1.5 , while in inter-urban studies, USAP [2] sets it as 1.6. When the problem extends to urban rail transit, the selection criteria of efficient paths are grouped into two categories as qualitative criteria and quantitative criteria. Both of them are based on the behavior assumption that passengers tend to choose the path with the shorter travel time and fewer times of interchange. Qualitative criteria consist of no repeated line in acyclic network, no repeated stations [3] and no roundabout interchanges [4]. All of them can be satisfied by adding judging conditions in the path search process to exclude inefficient paths. In quantitative criteria, more specific requirements for the cost (which is usually represented by travel time) and interchange times of efficient paths are raised, mainly including three points as follows:

1) Absolute threshold: If one path between its origin station $(\mathrm{O})$ and destination station (D) meets the requirement in eqn (1), then this path can be regarded as an efficient path.

$$
c \leqslant c_{\min }+f_{\max }
$$

where $c$ is the cost of one path between a certain OD, $c_{\min }$ is the cost of the shortest path between the OD, $f_{\max I}$ is the value of absolute threshold.

2) Relative threshold: If one path between its OD meets the requirement in eqn (2), then this path can be regarded as an efficient path.

$$
c \leqslant c_{\min }\left(1+f_{\max 2}\right)
$$

where $f_{\max 2}$ is the value of relative threshold. 
3) The limit of interchange times: If one path between its OD meets the requirement in eqn (3), then this path can be regarded as an efficient path.

$$
n \leqslant N_{\max }
$$

where $n$ is the interchange times of one path between a certain OD, $N_{\max }$ is the limit of interchange times.

Although the kind of quantitative criteria is quite similar in previous literature, the combinations and values are different (table 1).

Table 1: The combinations and values of efficient path selection criteria in literature.

\begin{tabular}{|c|c|c|c|c|c|c|}
\hline \multicolumn{2}{|c|}{ Classification } & Literature & \multicolumn{2}{|l|}{$\begin{array}{l}f_{\max 1} \\
(\min )\end{array}$} & $\begin{array}{c}f_{\max 2} \\
(\%)\end{array}$ & $\begin{array}{c}N_{\max } \\
\text { (times) }\end{array}$ \\
\hline \multirow{6}{*}{$\begin{array}{l}\text { Single } \\
\text { criterion }\end{array}$} & \multirow{4}{*}{$\begin{array}{l}\text { Only absolute } \\
\text { threshold }\end{array}$} & Si et al. [5] & 10 & & - & - \\
\hline & & \multirow{3}{*}{$\begin{array}{l}\mathrm{Su}[6] \\
\mathrm{Ma}[7]\end{array}$} & Short distance & 10 & \multirow{3}{*}{-} & \multirow{3}{*}{-} \\
\hline & & & $\begin{array}{l}\text { Medium } \\
\text { distance }\end{array}$ & 12 & & \\
\hline & & & Long distance & 15 & & \\
\hline & \multirow[t]{2}{*}{$\begin{array}{c}\text { Only relative } \\
\text { threshold }\end{array}$} & $\begin{array}{c}\text { Si et al. [8] } \\
\text { Liu [9] } \\
\text { Liu [4] } \\
\text { Qian et al. } \\
\text { [10] }\end{array}$ & \multicolumn{2}{|l|}{-} & 15 & - \\
\hline & & Yang [11] & \multicolumn{2}{|l|}{-} & 140 & - \\
\hline \multirow{8}{*}{$\begin{array}{l}\text { Multi- } \\
\text { criteria }\end{array}$} & \multirow{2}{*}{$\begin{array}{c}\text { Without a limit } \\
\text { to interchange } \\
\text { times }\end{array}$} & Luo [12] & 10 & & 60 & - \\
\hline & & $\mathrm{Li}[3]$ & \multicolumn{3}{|c|}{ Combined threshold } & - \\
\hline & \multirow{6}{*}{$\begin{array}{l}\text { With a limit to } \\
\text { interchange } \\
\text { times }\end{array}$} & \multirow{3}{*}{ Liu [13] } & Short distance & 10 & \multirow{3}{*}{-} & \multirow{3}{*}{2} \\
\hline & & & $\begin{array}{l}\text { Medium } \\
\text { distance }\end{array}$ & 12 & & \\
\hline & & & Long distance & 15 & & \\
\hline & & Guo [14] & \multicolumn{2}{|l|}{15} & 40 & 3 \\
\hline & & Kou [15] & \multicolumn{2}{|l|}{20} & 80 & 3 \\
\hline & & Pan [16] & \multicolumn{2}{|l|}{30} & 60 & 3 \\
\hline
\end{tabular}

Not all the paths searched according to selection criteria are actual efficient paths, but only those searched under rational criteria can be the basis of further study. So far, not much research has been done within the sensitivity analysis of selection criteria. By setting multiple combinations of absolute and relative threshold, $\mathrm{Li}$ [3] observes the number of efficient paths under various scenarios. It is found when one threshold increases to a certain value, the number will not change with the other threshold, which means that it reaches a stable state. The combination threshold at this time is chosen as the criteria. Liu [13] picks out three pairs of OD under short, medium and long distance respectively. By changing absolute threshold value and the limit of interchange times, the number of efficient paths and assignment results on each path are investigated. Results show that when the limit of interchange times increases from 2 to 3 , the number of efficient paths will rise, but the assignment results change little. However, if absolute threshold 
is too small, the second shortest path with high selection probability may be excluded from alternative efficient path sets. This may significantly affect assignment results. Liu [4] set relative thresholds as $0,50 \%$ and $100 \%$ and compares the estimated interchange passenger flow under them with actual survey data. When relative threshold equals to $50 \%$, average relative deviation is minimized. The above researches have contributed a lot, whereas several drawbacks still exist.

1) The aim of setting criteria is to obtain real efficient paths, but the number of efficient paths cannot give any information about the correctness. This makes it unreasonable to determine absolute and relative threshold values according to the stable state of efficient paths' number.

2) Interchange passenger flow is influenced by many factors, not only efficient paths, but also the form of impedance function as well as parameter values. So the deviation of passenger flow is unable to directly reflect which combination and value of selection criteria is better.

As stated above, there is no systematic and comprehensive sensitivity analysis of efficient path selection criteria. This study tries to make up for the deficiency.

\section{Study methodology}

\subsection{Data collection}

The mobile phone has become one of the most popular tools in people's daily life. With the support from network operators, mobile phone signalling data can be collected. The data contains location information of anonymous mobile phone users when they change base stations [17]. Every underground station in Shanghai metro network is covered by several specific base stations. When a passenger enters an underground station from street level or leaves it to go to street level, his/her mobile phone launches a location information report, from which the station and line information can be captured. The same situation occurs when a passenger interchanges between two underground stations. Thus the route choices of passengers become available. Thanks to the high ownership rate and usage of mobile phones within urban rail transit passengers, the sampling proportion of signalling data ranges from $10 \%$ to $25 \%$, which is much higher than the traditional resident trip survey, whose sampling proportion ranging from $2 \%$ to $5 \%$.

Sensitivity analysis needs passengers' route choice results between different OD pairs. These OD pairs must satisfy two requirements. The first one is that there are multiple alternative paths between the OD pair. Since mobile phone signalling data is only available for underground stations, the second one is that the shortest path between the OD pair must be an "underground" path. That is to say, the origin, destination and interchange stations must be underground stations.

This research develops an efficient path search program, with the application of a depth-first algorithm. A trial $\left(f_{\max }=10 \mathrm{~min}, f_{\max 2}=20 \%, N_{\max }=2\right)$ is conducted to find possible OD pairs. To ensure that the sample size is sufficient to support further analysis, 32 pairs of OD with considerable passenger flow are chosen as investigation objects. After the pre-processing of signalling data of Shanghai 
urban rail transit passengers on May 15th, 2013, 16,476 passengers' route choice results are derived. 7768 passengers travel forward (from $\mathrm{O}$ to $\mathrm{D}$ ), while 8708 passengers travel reversely (from $\mathrm{D}$ to $\mathrm{O}$ ).

\subsection{Sensitivity analysis method}

\subsubsection{The range of parameters}

To determine the range of threshold values for sensitivity analysis, the study groups all the samples by their absolute and relative differences between themselves and the shortest path of the OD pair, with a class interval of 2.5 minutes in absolute difference and 5\% in relative difference. The upper limit of subgroup is observed when cumulative frequency reaches $90 \%, 95 \%, 99 \%$ and $100 \%$ for the first time.

Table 2: The cumulative frequency of paths grouped by absolute difference.

\begin{tabular}{|c|c|c|c|c|}
\hline \multirow{2}{*}{$\begin{array}{c}\text { Cumulative } \\
\text { frequency } \\
\text { criterion (\%) }\end{array}$} & \multicolumn{2}{|c|}{ Forward journey } & \multicolumn{2}{c|}{ Reverse journey } \\
\cline { 2 - 5 } & $\begin{array}{c}\text { Upper } \\
\text { limit (min) }\end{array}$ & $\begin{array}{c}\text { Actual } \\
\text { cumulative } \\
\text { frequency (\%) }\end{array}$ & $\begin{array}{c}\text { Upper } \\
\text { limit (min) }\end{array}$ & $\begin{array}{c}\text { Actual } \\
\text { cumulative } \\
\text { frequency (\%) }\end{array}$ \\
\hline $\mathbf{9 0}$ & 5.0 & 95.37 & 5.0 & 94.06 \\
\hline $\mathbf{9 5}$ & 5.0 & 95.37 & 7.5 & 98.61 \\
\hline $\mathbf{9 9}$ & 12.5 & 99.45 & 12.5 & 99.49 \\
\hline $\mathbf{1 0 0}$ & 30.0 & 100.00 & 45.0 & 100.00 \\
\hline
\end{tabular}

Table 3: The cumulative frequency of paths grouped by relative difference.

\begin{tabular}{|c|c|c|c|c|}
\hline \multirow[b]{2}{*}{$\begin{array}{c}\text { Cumulative } \\
\text { frequency } \\
\text { criterion }(\%)\end{array}$} & \multicolumn{2}{|c|}{ Forward journey } & \multicolumn{2}{|c|}{ Reverse journey } \\
\hline & $\begin{array}{c}\text { Upper } \\
\operatorname{limit}(\%)\end{array}$ & $\begin{array}{c}\text { Actual } \\
\text { cumulative } \\
\text { frequency }(\%)\end{array}$ & $\begin{array}{c}\text { Upper } \\
\text { limit (\%) }\end{array}$ & $\begin{array}{c}\text { Actual } \\
\text { cumulative } \\
\text { frequency }(\%)\end{array}$ \\
\hline 90 & 10 & 91.57 & 10 & 90.40 \\
\hline 95 & 20 & 97.01 & 20 & 96.28 \\
\hline 99 & 40 & 99.29 & 35 & 99.02 \\
\hline 100 & 210 & 100.00 & 355 & 100.00 \\
\hline
\end{tabular}

From tables 2 and 3 , in either case, the cumulative frequency grows rapidly at first and slows down later. In other words, most passengers choose the paths whose difference are quite little. Nevertheless, passengers who choose the paths deviating from the shortest path a lot still exist. To prevent the interference caused by the small section of passengers, $90 \%$ and $99 \%$ are chosen as the criteria to determine the value range. The corresponding upper limits of subgroups are taken as the lower and upper limits respectively. So, absolute threshold ranges from 5.0 minutes to 12.5 minutes, and relative threshold ranges from $10 \%$ to $40 \%$.

\subsubsection{Two types of risks}

Values of selection criteria threshold directly affect the number and correctness of efficient paths. If the values are too low, the alternative efficient path set under the 
criteria may miss some actual efficient path. On the contrary, the set is likely to include some inefficient paths. To avoid these two cases, two types of errors should be observed when comparing threshold values.

The first type of error refers to the one where an alternative efficient path set hasn't included the path which is an actual efficient path. The probability of this error is called Risk I, shown by eqn (4).

$$
P\left(M_{1}\right)=\frac{|R|-\left|R \cap R^{\prime}\right|}{|R|}
$$

The second type of error refers to the one where an alternative efficient path set includes the path which isn't an actual efficient path. The probability of this error is called Risk II, shown by eqn (5).

$$
P\left(M_{2}\right)=\frac{\left|R^{\prime}\right|-\left|R \cap R^{\prime}\right|}{|R|}
$$

where $R$ is the actual efficient path set, $R^{\prime}$ is an alternative efficient set, and the symbol $|\cdot|$ means the number of elements in the set, which is equivalent to the number of paths here. According to their definitions, it is easy to find that with the rise of threshold values, Risk I decreases as Risk II increases. Although both risks are expected to reach the minimum simultaneously, it is hard to realize. So this paper proposes a new idea of sensitivity analysis, which is selecting the threshold value with a minimal sum of two types of risks as the best one, based on the premise that the alternative efficient path set under these criteria covers a certain percentage of passengers.

The first step of analysis is comparing the influence of absolute and relative threshold values on the size and two types of risks of alternative sets. For this goal, the limit of interchange times is kept as 2. Then, during the second phase, according to the development of an urban rail transit network, the limit of interchange times is set to be 2 and 3 respectively, with threshold values being the same as the first step. So the impact of interchange times can be observed.

\section{Sensitivity analysis}

\subsection{Absolute threshold}

Due to a slightly different run time and interchange time in two directions, the sizes of alternative efficient path sets of forward and reverse travels are not totally the same. But the growths of set sizes do not change significantly with the increase of absolute threshold (as stated in fig. 1). For every $2.5 \mathrm{~min}$ increment in absolute threshold, the number of forward efficient paths increases 23.0 medially, while the average increment of reverse efficient paths is 23.5 . It is worth noting that before the number reaches a stable state, the coverage of passenger flow has met $99 \%$, which means more inefficient paths may be included into alternative sets if the threshold value is determined according to the stable state.

The trend of the sum of two types of risks $\left(\sum R\right)$ depends on the changes of both. When absolute threshold increases, $\sum \mathrm{R}$ decreases at first, converses to rise later, 
and tends to be stable. When $f_{\max }=10.0 \mathrm{~min}, \sum \mathrm{R}$ of alternative efficient path sets in both directions reach the minimum, with the average value equal to $46.4 \%$. At this time, the coverages of forward and reverse passenger flows are $98.8 \%$ and $99.0 \%$. If higher precision is needed, taking $99 \%$ as the coverage criterion, the average sum is minimized when $f_{\max }=12.5 \mathrm{~min}$, which is $52.8 \%$.
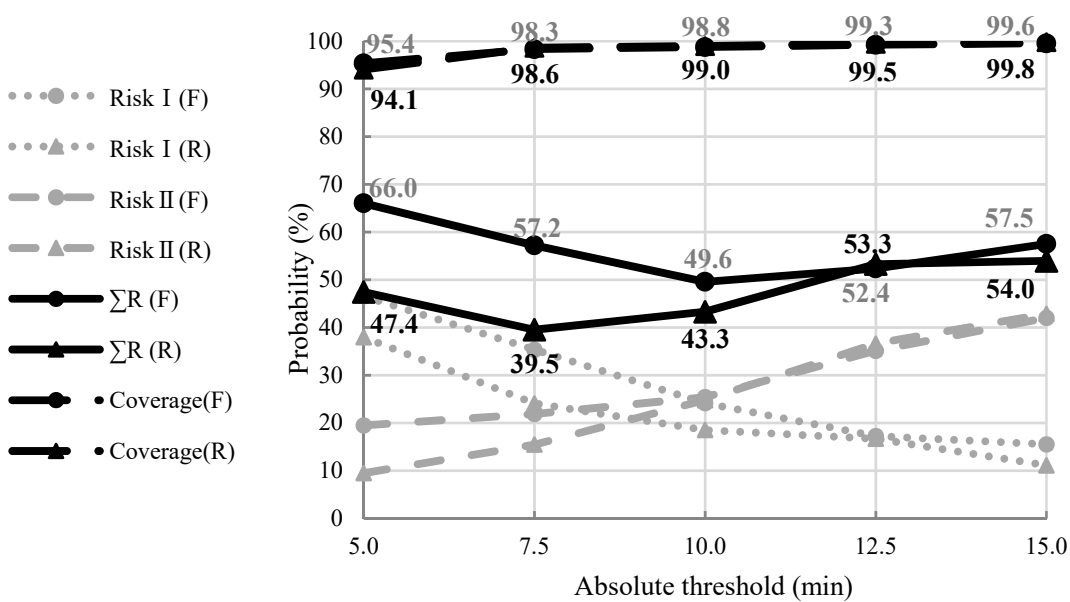

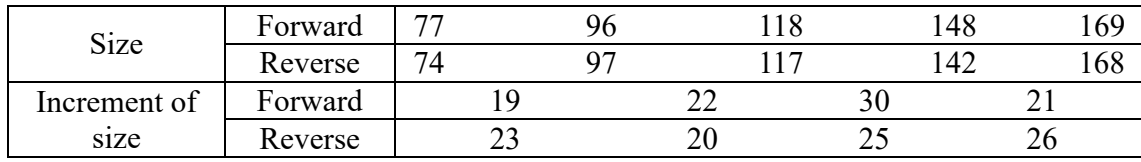

Figure 1: Statistics of alternative efficient path sets under various absolute threshold values $\left(\mathrm{N}_{\max }=2\right)$.

\subsection{Relative threshold}

Compared with absolute threshold, the number of efficient paths increases a little slower with the change of relative threshold value. For every 5\% increment in relative threshold, the number of forward efficient paths increases 12.8 medially, while the average increment of reverse efficient paths is 13.2. From fig. 2, when the coverage of passenger flows reaches $99 \%$, the size of alternative sets is still in the growth.

The trend of $\sum \mathrm{R}$ is similar to the one of absolute threshold. If the coverage criterion is $95 \%$, the average sum achieves the minimum $47.9 \%$ when $f_{\text {max }}=35 \%$. With the improvement of accuracy, taking $99 \%$ as the coverage criterion, the least value is attained as $f_{\max 2}=40 \%$ and the average sum is equal to $51.6 \%$.

Based on the passengers' route choices of 32 OD pairs, with the limit of interchange times being kept as 2, absolute threshold has a better performance when the coverage criterion is $95 \%$, while relative threshold performs better when the criterion is $99 \%$. 

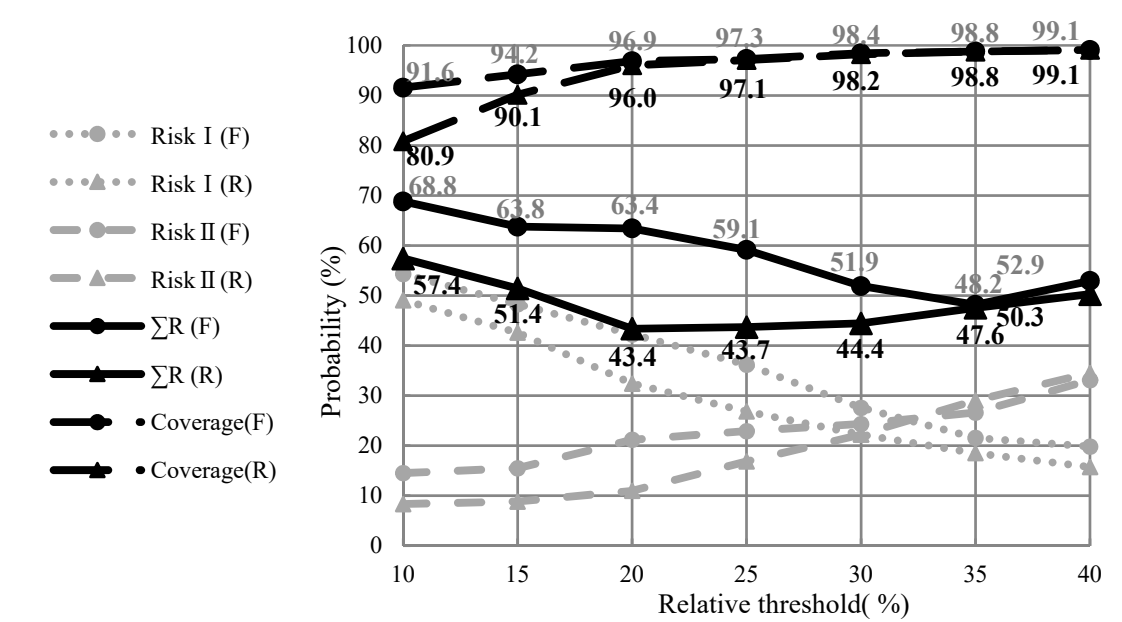

\begin{tabular}{|c|c|ccccccccc|}
\hline \multirow{2}{*}{ Size } & Forward & 62 & 71 & 85 & 96 & 111 & 124 & 139 \\
\cline { 2 - 11 } & Reverse & 60 & 68 & 82 & 95 & 108 & 124 & 139 \\
\hline $\begin{array}{c}\text { Increment } \\
\text { of size }\end{array}$ & Forward & 26 & & 34 & 58 & 54 & 26 & 34 \\
\cline { 2 - 11 } & Reverse & 31 & & 26 & 60 & 61 & 31 & 26 \\
\hline
\end{tabular}

Figure 2: Statistics of alternative efficient path sets under various relative threshold values $\left(\mathrm{N}_{\max }=2\right)$.

\subsection{The limit of interchange times}

When the limit of interchange times rises from 2 to 3 , the sizes of alternative sets become much larger, whereas the coverages are approximately the same. For every $2.5 \mathrm{~min}$ increment in absolute threshold, the average increment of forward efficient paths number is 43.0, and the one of reverse efficient paths number is 44.5. The speed when $\mathrm{N}_{\max }=3$ is 1.87 times and 1.89 times as the ones when $\mathrm{N}_{\max }=2$. For every $5 \%$ increment in relative threshold, the average increment of forward efficient paths number is 24.8 , while the one of reverse efficient paths number is $25.7,1.94$ times and 1.95 times as the ones when $\mathrm{N}_{\max }=2$. Meanwhile, the increment increases with the expansion of threshold. The reason is that when thresholds increase, there are much more feasible paths between two stations in a network. The relaxed limit of interchange times allows more paths into the alternative sets.

Although the coverage is close, the sum of two types of risks when $\mathrm{N}_{\max }=3$ is much higher, whether under absolute or relative threshold. The differences between $\Sigma \mathrm{R}$ when $\mathrm{N}_{\max }=2$ and 3 in both directions are widened with the swell of thresholds. It is mainly because the validity of paths included into the alternative sets due to the increase of the limit is much lower than those in the original sets. This will lead to the rapid growth of Risk II. As a result, taking 95\% and 99\% as the coverage criterion, when $\Sigma \mathrm{R}$ reaches minimum, absolute threshold values are totally the same as the ones when $\mathrm{N}_{\max }=2$, but the minimal average sums are higher, $6.8 \%$ (coverage criterion $=95 \%$ ) and $11.8 \%$ (coverage criterion $=99 \%$ ) respectively 
(fig. 3(a)). When it comes to relative threshold, taking $95 \%$ as the coverage criterion, the minimum is achieved when $f_{\max 2}=20 \%$, with the average sum being $59.7 \%, 11.8 \%$ higher than the one when $\mathrm{N}_{\max }=2$. Taking $99 \%$ as the criterion, the minimum is achieved when $f_{\max 2}=40 \%$, which is $71.2 \%, 19.6 \%$ higher (fig. $3(\mathrm{~b})$ ).
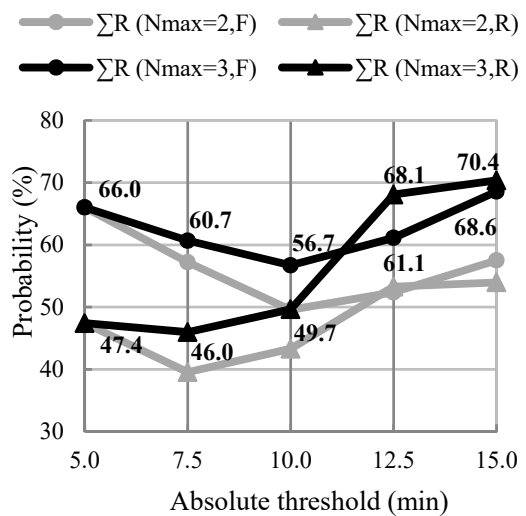

(a)

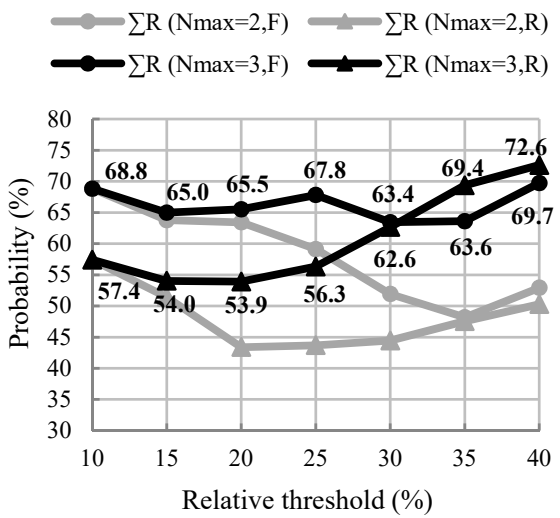

(b)

Figure 3: The sum of two types of risks under different thresholds.

Analyses show that if the limit of interchange times is 3, a large number of inefficient paths would be included. On the other hand, if the limit is 2 , efficient paths of some OD pairs would be missed when the search scope extends to the whole network, on account of the fact that there must be some long-distance journeys, of which the shortest paths need 3 times interchange. Thus a fixed limit of interchange times is likely to lead to the distortion of alternative efficient path sets.

\section{Optimization of efficient path selection criteria}

\subsection{Threshold combination}

For passengers travelling different distances, the accepted thresholds are not all the same. This cannot be described well by single threshold, neither absolute threshold nor relative threshold. So the paper proposes adopting a threshold combination instead of a single threshold.

Absolute threshold ranging from $5.0 \mathrm{~min}$ to $15.0 \mathrm{~min}$ with the interval of $2.5 \mathrm{~min}$, relative threshold ranging from $10 \%$ to $60 \%$ and the limit of interchange times set as 2 and 3 are combined to be the selection criteria. Two types of risks are investigated under the $95 \%$ and $99 \%$ coverage. The best combinations under distinct limits of interchange times are shown in table 4.

Under the same requirement of coverage, the alternative efficient path set under the best threshold combination has a better performance than the one under the best single threshold, no matter how much the limit of interchange times is. It is 
proved that threshold combination is superior to a single threshold, owning to its ability to reduce two types of risks effectively while ensuring the coverage of passenger flow.

Table 4: The best threshold combinations under different coverage criteria.

\begin{tabular}{|c|c|c|c|c|c|}
\hline \multirow{2}{*}{\multicolumn{2}{|c|}{$\frac{\text { Coverage criterion }(\%)}{f_{\max 1}(\mathrm{~min})}$}} & \multicolumn{2}{|c|}{95} & \multicolumn{2}{|c|}{99} \\
\hline & & 10.0 & 10.0 & 15.0 & 12.5 \\
\hline \multicolumn{2}{|c|}{$f_{\max 2}(\%)$} & 35 & 50 & 40 & 55 \\
\hline \multicolumn{2}{|c|}{$N_{\max }$ (times) } & 2 & 3 & 2 & 3 \\
\hline \multirow{2}{*}{ Actual coverage $(\%)$} & Forward & 98.7 & 98.8 & 99.1 & 99.2 \\
\hline & Reverse & 98.7 & 99.0 & 99.1 & 99.0 \\
\hline \multirow{2}{*}{ Risk I (\%) } & Forward & 25.9 & 22.4 & 20.7 & 13.8 \\
\hline & Reverse & 21.3 & 17.6 & 15.7 & 16.7 \\
\hline \multirow{2}{*}{ Risk II (\%) } & Forward & 23.2 & 33.8 & 32.4 & 46.8 \\
\hline & Reverse & 19.0 & 31.5 & 33.1 & 51.1 \\
\hline \multirow{2}{*}{$\sum \mathbf{R}(\%)$} & Forward & 49.1 & 56.2 & 53.0 & 60.6 \\
\hline & Reverse & 40.3 & 49.1 & 48.8 & 67.8 \\
\hline \multicolumn{2}{|c|}{$\begin{array}{l}\text { The average sum under this } \\
\text { threshold combination }(\%)\end{array}$} & 44.7 & 52.7 & 50.9 & 64.2 \\
\hline \multirow{2}{*}{$\begin{array}{c}\text { The minimal average } \\
\text { sum under single } \\
\text { threshold }(\%)\end{array}$} & $\begin{array}{l}\text { Absolute } \\
\text { threshold }\end{array}$ & 46.4 & 53.2 & 52.8 & 64.6 \\
\hline & $\begin{array}{l}\text { Relative } \\
\text { threshold }\end{array}$ & 47.9 & 59.7 & 51.6 & 71.2 \\
\hline
\end{tabular}

When the coverage criterion is $95 \%$, the alternative efficient path set under the threshold combination where $f_{\max I}=10.0 \mathrm{~min}, f_{\max 2}=35 \%, N_{\max }=2$ performs the best. In this case, for the OD pairs of which the shortest path costs less than 28.6 minutes, relative threshold works. For the remaining OD pairs, passengers choose efficient paths according to absolute threshold; that is to say, the accepted time difference is 10 minutes. When the coverage criterion is $99 \%$, the alternative set under the threshold combination where $f_{\max }=15.0 \mathrm{~min}, f_{\max 2}=40 \%, N_{\max }=2$ reaches the minimum. The dividing line of absolute and relative threshold increases to 37.5 minutes. In spite of this it still stabilizes at around 30 minutes.

\subsection{The limit of interchange times based on the shortest path}

For the whole urban rail transit network, searching efficient paths under a fixed limit of interchange times is prone to distort alternative sets. To solve the problem, the paper tries a new approach to determine the limit of interchange times, based on the assumption that passengers prefer to choose paths with shorter travel and interchange times. The approach is adjusting the limit of interchange times according to the shortest path's interchange times of the same OD pair, instead of a fixed number. Combined with single thresholds and threshold combinations, the best alternative efficient path sets under various scenarios are shown in table 5. 
Table 5: The best thresholds under the limit of interchange times based on the shortest path.

\begin{tabular}{|c|c|c|c|c|}
\hline \multirow{2}{*}{\multicolumn{2}{|c|}{$\frac{\text { Coverage criterion }(\%)}{f_{\max }(\mathrm{min})}$}} & \multicolumn{3}{|c|}{95} \\
\hline & & 10.0 & - & 10.0 \\
\hline \multicolumn{2}{|c|}{$f_{\max 2}(\%)$} & - & 35 & 45 \\
\hline \multirow{2}{*}{ Actual coverage $(\%)$} & Forward & 98.2 & 98.2 & 98.2 \\
\hline & Reverse & 98.3 & 98.1 & 98.1 \\
\hline \multirow{2}{*}{ Risk I (\%) } & Forward & 37.9 & 36.2 & 37.9 \\
\hline & Reverse & 27.8 & 26.9 & 28.7 \\
\hline \multirow{2}{*}{ Risk II (\%) } & Forward & 16.3 & 19.6 & 16.3 \\
\hline & Reverse & 8.2 & 13.2 & 7.2 \\
\hline \multirow{2}{*}{$\sum \mathbf{R}(\%)$} & Forward & 54.2 & 55.8 & 54.2 \\
\hline & Reverse & 36.0 & 40.0 & 35.9 \\
\hline \multicolumn{2}{|c|}{$\begin{array}{c}\text { The average sum under changing } \\
\text { limit of interchange times }(\%)\end{array}$} & 45.1 & 47.9 & 45.1 \\
\hline \multirow{2}{*}{$\begin{array}{c}\text { The minimal average } \\
\text { sum under fixed } \\
\text { limit of interchange } \\
\text { times }(\%)\end{array}$} & $N_{\max }=\mathbf{2}$ & 46.4 & 47.9 & 44.7 \\
\hline & $N_{\max }=\mathbf{3}$ & 53.2 & 59.5 & 52.7 \\
\hline
\end{tabular}

The results in table 5 suggest that only when combined with a single threshold can the new approach bring Risk II down. Even Risk I slightly lifts, the minimal $\Sigma \mathrm{R}$ is lower than the one under a fixed limit. While the alternative set under changing limit and threshold combination plays much better than the one when $N_{\max }=3$, but it is inferior to the one when $N_{\max }=2$. Some of the reasons for this are the shortest paths' interchange times of 32 investigated OD pairs are no more than 2 times. The effect of the new approach is weakened to some extent. For those OD pairs of which the shortest path's interchange times are more than 2, the applicability of this approach needs to be verified by supporting data.

The final search results have demonstrated that the limit of interchange times based on the shortest path can prevent many inefficient paths entering alternative sets, but at the same time, it can lead to missing a portion of actual efficient paths. That's why the alternative sets under the changing limit cannot cover more than 99\% of passengers. Passengers choose the missed paths mainly because they cannot afford the crowding of shorter paths. The missed paths are more comfortable, but they are not as good as the paths in alternative sets in terms of travel time and interchange times.

\section{Conclusions}

A systematic sensitivity analysis is conducted in the paper. Before the number of efficient paths stabilizes, the coverage of passenger flow has reached $99 \%$. So the determination of absolute and relative thresholds cannot rely on the change of number of efficient paths. Otherwise, a large number of inefficient paths would be 
included into alternative sets. Instead, two types of risks are proposed to analyse the influence of threshold value changes.

To optimize efficient path selection criteria, threshold combination and adjusting limit of interchange times based on the shortest path are suggested. Results show that no matter how much the coverage is or how many the limit of interchange times is, the best alternative efficient path sets under threshold combination has a better performance than the best one under a single threshold. On the other side, the limit of interchange times based on the shortest path can effectively reduce Risk II of alternative sets, with the best coverage being no less than $98 \%$. But it is not suitable for a highly crowded urban rail transit network, as the proportion of passengers choosing a more comfortable path with more interchange instead of a less comfortable path with less interchange would rise.

The paper yields the best threshold values under various scenarios. Compared with this, absolute threshold values in previous studies are a little higher while relative threshold values are a bit lower if single threshold is adopted. In the case of combined thresholds, both values are higher.

\section{References}

[1] Tagliacozzo, F. \& Pirzio, F., Assignment models and urban path selection criteria: Results of a survey of the behaviour of road users. Transportation Research, 7(3), pp. 313-329, 1973.

[2] USAP, Comportement des automobilistes en matière de choix d'itinéraires. Research report, USAP: Paris, 1992.

[3] Li, J.H., Study on the passenger flow assignment model and algorithm of urban rail transit network, M.S. thesis, Beijing Jiaotong University: Beijing, 2010.

[4] Liu, X.H., Research on the dynamic flow assignment model based on train schedule for urban subway network, Ph.D. thesis, Chang'an University: Xi'an, 2013.

[5] Si, B.F., Mao, B.H. \& Liu, Z.L., Passenger flow assignment model and algorithm for urban railway traffic network under the condition of seamless transfer. Journal of the China Railway Society, 29(6), pp. 12-18, 2007.

[6] $\mathrm{Su}, \mathrm{J}$., Research on passenger flow assignment of urban rail transit, M.S. thesis, Beijing Jiaotong University: Beijing, 2009.

[7] Ma, F., Research on passenger flow assignment of urban rail transit, M.S. thesis, Southwest Jiaotong University: Chengdu, 2009.

[8] Si, B.F., Zhang, H.Z. \& Gao, Z.Y., Improved Dial's algorithm for Logitbased stochastic traffic network assignment problem. China Journal of Highway and Transport, 22(1), pp. 78-83, 2009.

[9] Liu, J.F., Transfer-based passenger flow assignment model of urban rail transit and empirical research, Ph.D. thesis, Beijing Jiaotong University: Beijing, 2012.

[10] Qian, K., Chen, Y.\& Mao, B.H., Route choice behavior for urban rail transit considering transfer time. Journal of Transportation Systems Engineering and Information Technology, 15(2), pp. 116-121, 2015. 
[11] Yang, D.C., Research on schedule-based rail transit passenger flow assignment, Ph.D. thesis, Beijing Jiaotong University: Beijing, 2013.

[12] Luo, Q., Research on passenger flow assignment theory and simulation for urban rail transit based on network operations, Ph.D. thesis, Tongji University: Shanghai, 2009.

[13] Liu, G.Y., Research on ticket income distribution problem in urban rail transit system network, M.S. thesis, Central South University: Changsha, 2011.

[14] Guo, Y.Y., Research on efficient paths for urban rail transit, M.S. thesis, Beijing Jiaotong University: Beijing, 2011.

[15] Kou, T.T., Research on the distribution of tickets income from subway network, M.S. thesis, Southwest Jiaotong University: Chengdu, 2010.

[16] Pan, X., Research on income distribution model of urban rail transit under the network condition, M.S. thesis, Southwest Jiaotong University: Chengdu, 2011.

[17] Ran, B., Use of cell phone data in travel survey and transportation planning. Urban Transport of China, 11(1), pp. 72-81, 2013. 